\title{
Experimental and numerical study of crack damage under variable amplitude thermal fatigue for compacted graphite iron EN-GJV-450
}

\author{
Sining Pan ${ }^{\mathrm{a}, \mathrm{b}}$, Gang $\mathrm{Yu}^{\mathrm{a}, \mathrm{b}, *}$, Shaoxia $\mathrm{Li}^{\mathrm{a}}$, Xiuli $\mathrm{He}^{\mathrm{a}, \mathrm{b}, *}$, Ru Chen ${ }^{\mathrm{a}, \mathrm{b}}$ \\ a Institute of Mechanics, Chinese Academy of Sciences, China \\ ${ }^{\mathrm{b}}$ School of Engineering Science, University of Chinese Academy of Sciences, China
}

\section{A R T I C L E I N F O}

\section{Keywords:}

Crack damage

Variable amplitude thermal fatigue

Surface wedging process

Cast iron

Combustion chamber

\begin{abstract}
A B S T R A C T
Fatigue crack under variable amplitude thermal cycles is a common failure in combustion chamber components, proposing great challenges during the temperature reproduction of actual working condition. In this paper, this complicated thermal cycle was realized with pulsed laser experimentally, causing two kinds of damage such as the low cycle thermal fatigue (LCF) and high cycle thermal fatigue (HCF). A numerical model was developed to simulate the temperature, stress and strain under variable amplitude thermal cycles. The results showed that, there was good agreement between the measured temperature curve and the simulated results with different crack parameters. The observed crack depth at different cycles was consistent with the predicted ones. Furthermore, the mechanism of crack evolution under variable amplitude thermal fatigue was discussed. The calculated thermal-structural interaction depth of LCF thermal cycle was found to be larger than the HCF thermal cycle. The effect of LCF thermal loadings was associated with the initiation of major cracks, while the failure related to the superimposed HCF action was the acceleration of crack growth with a surface wedging process. This paper provides comprehensive experimental and numerical insights into the thermal damage process under variable amplitude thermal loading, showing a significant engineering value for failure analysis and design optimization of combustion chamber components.
\end{abstract}

\section{Introduction}

Thermal cracking is a typical failure mode of high temperature combustion chamber components used in power plant, aerospace and transportation industry [1-3]. The crack damage caused by complicated thermal loading is becoming more and more serious with the development of high performance and reliability. The combined working conditions of combustion chamber components include startstop cycles and routine working cycles simultaneously [4-7]. The former ones refer to larger temperature range (change from $100^{\circ} \mathrm{C}$ to $600^{\circ} \mathrm{C}$ ) with a time period in several hundred seconds, which usually cause the low cycle fatigue (LCF) damage. In comparison, the latter ones correspond to smaller temperature range (the amplitude is $20-80^{\circ} \mathrm{C}$ ) with a time period in the order of milliseconds, which induce the high cycle fatigue (HCF) damage. The pure LCF or HCF tests consist of constant amplitude cyclic loading. In contrast, the combination of LCF with HCF leads to complex failure mode called combined cycle fatigue (CCF), which is subjected to variable amplitude cyclic loading essentially. As a result, the crack damage caused by the CCF tests is supposed to be different from that caused by pure LCF or HCF tests [8].
In the component design stage, several heat sources have been applied for the thermal fatigue reliability test, such as high frequency induction [9], quartz lamp [10], continuous wave laser [11,12] and so on. However, the thermal HCF superimposed within millisecond interval is hard to accomplish with the above methods. Therefore, the pulsed laser is considered to be the ideal heat source for the thermal fatigue reliability test of variable amplitude thermal cycles $[3,13,14]$, owing to its high temporal and spatial controllability. Both the LCF thermal shock and the HCF temperature oscillation can be realized by appropriate selection of pulsed parameters. The instantaneous and inhomogeneous temperature gradients induce large deformation between the surface and interior, and cause irreversible thermal damage. Once the damage accumulates to a certain extent, thermal fatigue crack will be produced finally. It is worth noting that, during the CCF testing process of variable amplitude loads, the direction of principal stress varies with cyclic loading, and is time-dependent. In addition, the amplitude of principal stress may no longer be proportional to one another. The above two factors indicate that the materials are under multiaxial fatigue condition, and the resulting CCF thermal cracks are supposed to be different from that caused by uniaxial fatigue.

\footnotetext{
* Corresponding authors at: Institute of Mechanics, Chinese Academy of Sciences, China.

E-mail addresses: gyu@imech.ac.cn (G. Yu), xlhe@imech.ac.cn (X. He).
} 
As for the recent researches carried out with the pulsed laser, Schaus [13] employed Nd:YAG pulsed laser to simulate thermal damage of steel as a result of cyclic temperature gradients. Long [15] studied the thermal fatigue of particle reinforced metal-matrix composite induced by pulsed laser heating and mechanical load, and simulated the fields and histories of temperature, macroscopical stress and microscopical stress with finite element software. Zhu [2] focused on the thermal barrier coating systems under thermal CCF conditions for diesel engine application, and the mechanisms of fatigue crack initiation and propagation. Vincent [16] proposed a thermal fatigue testing facility conducted with a pulsed laser beam, and an estimation of strain change was done using Digital Image Correction techniques. Although it has been reported above that, stresses generated by pulsed laser can initiate surface cracks, the detailed mechanisms of the crack evolution under the complex LCF and HCF conditions are still not completely understood and analyzed quantitatively.

During the pulsed laser CCF thermal cycles, the surface cracks initiate and propagate gradually. This time-dependent change influents the absorption of input energy, thus affecting the whole process of thermal deformation in a coupled and nonlinear way [17]. In this paper, the pulsed laser was applied for realizing the specific spatial and temporal temperature distribution, in order to reproduce the CCF thermal process. A nonlinear transient finite element analysis was conducted for the simulation of temperature and stress. Qualitative and quantitative analyses of CCF thermal cracks were conducted to investigate the effect of superimposed HCF loads on the thermal damage evolution of cast iron materials. Based on the above results, the mechanism of crack initiation and propagation under variable amplitude thermal fatigue was discussed. The present study provides insights into the variable amplitude thermal fatigue experimentally and numerically, which can help to analyze the thermal cracking failure and proposing evaluation criteria of combustion chamber components.

\section{Experimental procedure}

The investigated material is GJV-450 (compacted graphite) cast iron, which is one of the typical materials used in the cylinder head of diesel engine. As the microstructure showed in Fig. 1, 85\% of the graphite phase is vermicular and some spherical graphite phases scatter in the matrix. The graphite is with average length of $70-100 \mu \mathrm{m}$, and the width of $10 \mu \mathrm{m}$. The specimens are cut into cylindrical pieces with the size of $\Phi 18 * 5 \mathrm{~mm}$.

The experiment is carried out with self-designed testing apparatus [18]. A Nd:YAG millisecond pulsed laser with wavelength of $1.064 \mu \mathrm{m}$ is utilized as the heat source in this study. The pulsed laser energy is measured with Ophir-NOVA energy meter. The output pulse waveform

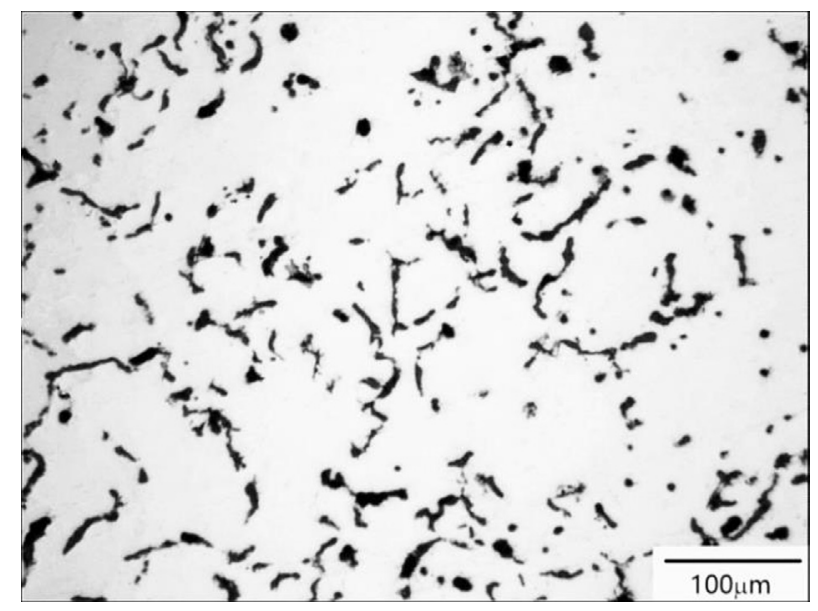

Fig. 1. Typical microstructure of GJV-450 material (un-etched). is set to be rectangular wave, and monitored by oscilloscope. The energy distribution of laser beam shows a super-Gaussian profile on the irradiated surface. Appropriate defocusing distance is selected for the laser spot diameter of $6 \mathrm{~mm}$. Temperature in the center of the laser beam is detected by infrared pyrometer (with wavelength of $8-14 \mu \mathrm{m}$ ), and the corresponding measurement error is $0.5 \%$. The detected temperature signal is important during the test, not only for the characterization of the thermal behavior, but also for the testing control. Compressed air serves as the cooling medium, suppling airflow to the backside of specimen through a gas nozzle, for maintaining the desired temperature gradients across the sample.

In order to produce the variable amplitude thermal cycles, a complete testing cycle is designed to include three stages: the LCF fast heating stage, the HCF temperature oscillation stage and the fast cooling stage, as illustrated in Fig. 2 schematically. The first stage is conducted by PC programmed software with temperature-controlled mode, which the maximal temperature $\mathrm{T}_{\max }$ is set as $450^{\circ} \mathrm{C}$. According to our previous research [18], the laser parameters of higher repetition rate are used in the first stage, such as $20 \mathrm{~Hz}$. After reaching the maximum temperature $T_{\max }$, the second stage is going on with time-controlled mode, which the heating time is fixed to $250 \mathrm{~s}$. At the same time, the laser parameters are switched to lower repetition rate in order to induce stable oscillation, such as $4 \mathrm{~Hz}$ for the cast iron specimen with a characteristic thickness of $5 \mathrm{~mm}$. Once the above two heating stages have been finished, the pulsed laser is turned off and the compressed air is blown to the backside of specimen until the detected temperature dropped to the minimal temperature $\mathrm{T}_{\min }$ of $100^{\circ} \mathrm{C}$. Since the three parts have been executed, a cycle block is finished. The surface cracks evolution is observed after the specific cycles with optical microscope $(\mathrm{OM})$ and scanning electron microscopy (SEM), in order to verify the numerical results.

\section{Numerical model}

Temperature data are the foundation of assessing thermal stress and thermal strain. However, it is difficult to measure the overall temperature of specimen. Therefore, the proposed model is to describe the thermal-structural coupling process of surface cracked body under variable amplitude thermal loading, which is based on the nonlinear thermal transient finite element analysis considering the heat conduction, convection and radiation effects (shown in Fig. 3(a)). The changing crack parameters induced by repeated heating of pulsed laser is considered in the geometric model. The evolution of crack numbers, width and length can be determined by surface observation easily, however, the growth of crack depth is unknown. In order to characterize the in-depth evolution of surface cracks (still within the thin layer below the surface), a two-dimensional axisymmetric model of longitudinal section is established (shown in Fig. 3(b)), with the same width and depth of each crack for simplification. The material is assumed as homogenous and the material parameters are listed in Table 1.

The temperature field due to the absorbing pulsed laser energy is governed by the Fourier equation [19]

$\rho C \frac{\partial T}{\partial t}-k \nabla^{2} T=Q$

where $\rho, C$ and $\mathrm{k}$ are the density, specific heat capacity and thermal conductivity respectively. $\mathrm{Q}$ is a volumetric heat source within the sample, which can be neglected in the model. A comprehensive heat transfer coefficient is used for simplification of radiation and convection with the environment. According to Fourier's heat conduction law, the boundary conditions on the top surface (including the cracked zone and the un-cracked zone) during the pulse duration obeyif (i -1$)$. $(\mathrm{w}+\mathrm{v})+\mathrm{w}<\mathrm{x}<\mathrm{i} \cdot(\mathrm{w}+\mathrm{v})$, and $\mathrm{i}=1 \sim \mathrm{n}$, then 


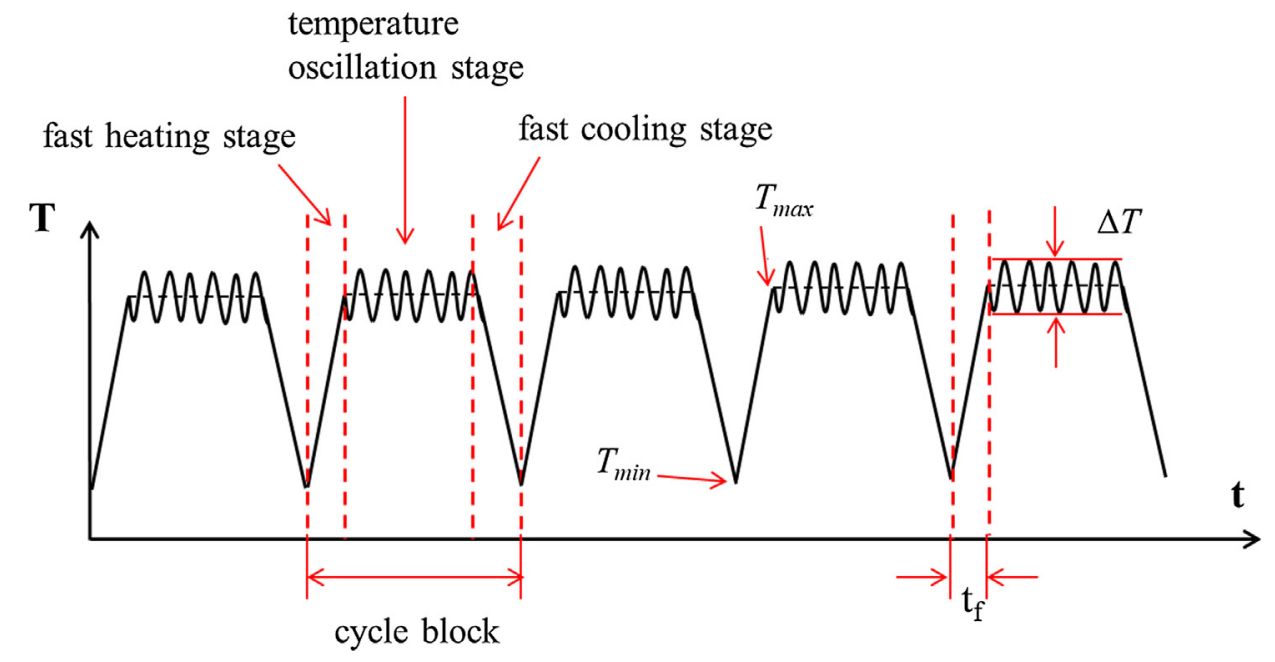

Fig. 2. Schematic diagram of the testing cycle.

$-\left.k \frac{\partial T}{\partial y}\right|_{y=0}=\alpha(T) I(x)$

if $(\mathrm{i}-1) \cdot(\mathrm{w}+\mathrm{v}) \leq \mathrm{x} \leq(\mathrm{i}-1) \cdot(\mathrm{w}+\mathrm{v})+\mathrm{w}$, and $\mathrm{i}=1 \sim \mathrm{n}$, then

$-\left.k \frac{\partial T}{\partial y}\right|_{y=b}=\alpha(T) I(x)$

$I(x)=I_{0} \exp \left[-2\left(\frac{x}{R}\right)^{N}\right]=\frac{E}{\pi R^{2} \tau} \exp \left[-2\left(\frac{x}{R}\right)^{9}\right]$

where $\alpha(T)$ is the laser absorptivity which depends on both material and temperature, and the spatial distribution of pulsed laser is assigned as $\mathrm{I}(\mathrm{x})$ with the super-Gaussian distribution $(\mathrm{N}=9) . \mathrm{n}$ is the crack number, while the crack width, crack interval, and crack depth is $\mathrm{w}, \mathrm{v}$ and $\mathrm{b}$ respectively. The radius and thickness of model is marked as $\mathrm{R}$ and $H$ respectively. E and $\tau$ represents the single pulse energy and pulse duration separately.

(2)
Table 1

Properties of GJV-450 material for simulation.

\begin{tabular}{lllllll}
\hline $\mathrm{T}(\mathrm{K})$ & $\mathrm{k}(\mathrm{W} / \mathrm{m} * \mathrm{~K})$ & $\mathrm{C}(\mathrm{J} / \mathrm{kg} * \mathrm{~K})$ & $\rho\left(\mathrm{kg} / \mathrm{m}^{3}\right)$ & $\mathrm{v}$ & $\alpha\left(10^{-6} / \mathrm{K}\right)$ & $\mathrm{E}(\mathrm{GPa})$ \\
\hline 300 & 43.0 & 441 & 7079 & 0.25 & $/$ & 106 \\
400 & 41.9 & 470 & & & 9.61 & 101 \\
500 & 40.2 & 513 & & 11.7 & 92.3 \\
600 & 38.6 & 562 & & 12.7 & 83.8 \\
700 & 36.4 & 615 & & 13.3 & 77.3 \\
800 & 33.8 & 674 & & 13.9 & 71.2 \\
\hline
\end{tabular}

Once the pulsed laser duration is finished, the top surface is under natural convection condition $\left(20 \mathrm{~W} / \mathrm{m}^{2} * \mathrm{~K}\right)$, while the back surface is forced convective with the environment $\left(100 \mathrm{~W} / \mathrm{m}^{2} * \mathrm{~K}\right)$. In addition, the adiabatic boundary conditions are set for the symmetric planes and the side surface. With regard to the significant temperature change on the specimen surface and large thermal gradient across the thickness,

(a)

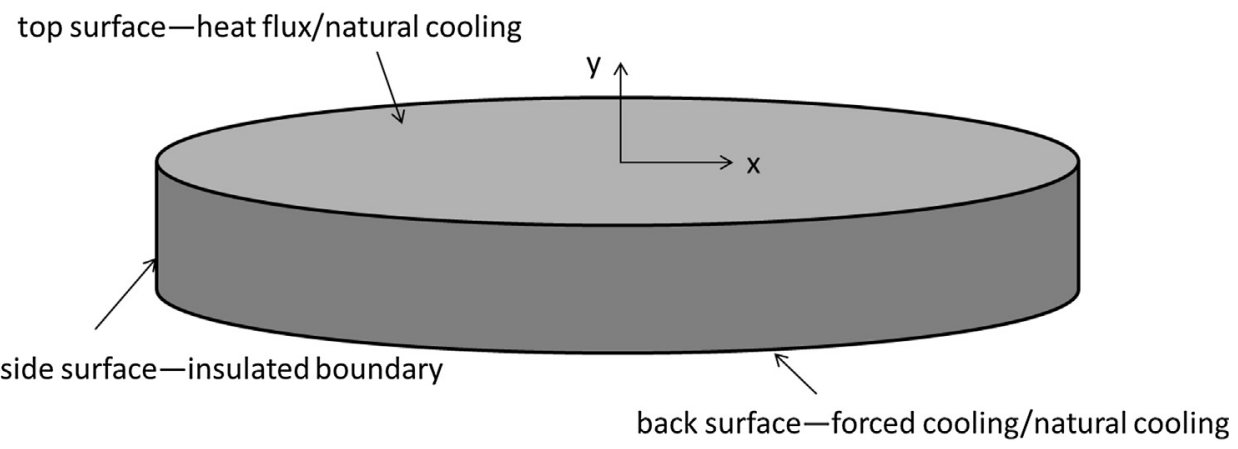

(b) laser beam radius

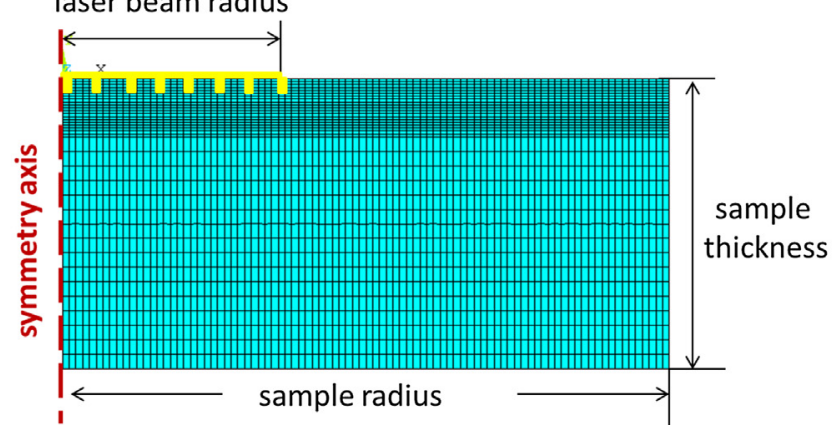

Fig. 3. Schematic description of (a) geometric model and boundary conditions; (b) meshes. 
the mesh size of near $1 \mathrm{~mm}$ depth from the surface is smaller than the bottom's, and the mesh model and the heat flux loading area is shown in Fig. 3b). The meshes are 28,350, and the grid independence is verified with an invariance of simulated temperature field.

The mechanical analysis is conducted using the temperature field results for loading conditions. The model is free of constraint and the symmetrical displacement boundary condition is applied to the symmetric axis.

\section{Results and analysis}

Thermal fatigue in combustion chamber components is a very complex process. The pulsed laser tests can capture some main features, such as initiation by a network of surface cracks, and propagation of many cracks that often arrest at some depth. Whether cracks remain arrest at a certain depth or propagate to be through-wall cracks depends mainly on the loading and the component geometry [20]. In order to focus on the effect of combined thermal cycles with variable amplitude and frequency, no additional mechanical constraint is applied to the specimen. Therefore, only surface cracks within a thin layer are induced and studied.

There are two important thermal indicators describing the combined thermal process, i.e. the LCF fast heating time $\left(t_{f}\right)$ and the HCF temperature oscillation range $(\Delta T)$, as shown in Fig. 2. They are closely related to the crack propagation on the substrate surface. The evolution curves of the two indicators in the experiment are presented in Fig. 4. Since the same preset stop temperature of $450^{\circ} \mathrm{C}$ in the first stage, as the cycles going, the $t_{f}$ decreases sharply from $39 \mathrm{~s}$ to $13 \mathrm{~s}$ at the first 10 cycles. After that, the descent rate becomes smaller, and the final value of 40 th cycle is $8 \mathrm{~s}$. Moreover, although the input energy during the second stage keeps the same value in every cycle, exponentially increased $\Delta T$ is observed. This increasing tendency slows down after 10 cycles, and reaching the maximal value of $60^{\circ} \mathrm{C}$ in the 40 th cycle. Such phenomena in the heat transfer process are associated with the change of surface condition, to be exactly, the surface cracks evolution. During the tests, the nonlinear change of absorptivity is significant, especially when the surface cracks are formed within the irradiated region. The laser beam will generate multiple reflections in the grooves of the cracked surface, thus being absorbed more. The more and deeper cracks occurred, the more energy is absorbed, making the fast heating stage shorter and larger temperature swing. Therefore, the proposed thermal simulation model is based on the above physical effect.

The analysis of crack parameters is performed in order to demonstrate the relation between the crack features and the thermal indicators. The effect of cracks evolution on the thermal process is characterized by the crack depth $\mathrm{b}$ and crack numbers $\mathrm{n}$. As for the vermicular cast iron material, the thermal cracks usually initiate at the graphite tip, and grow along the graphite phase [8]. Therefore, the

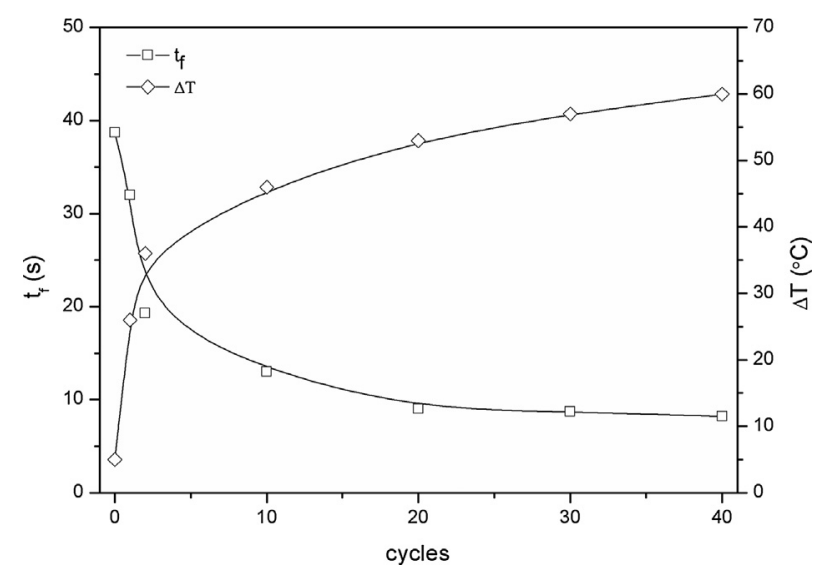

Fig. 4. Variation of $\mathrm{t}_{\mathrm{f}}$ and $\Delta \mathrm{T}$ with cycles. crack width $\mathrm{w}$ is approximately the same with the width of vermicular graphite, where the constant value of $10 \mu \mathrm{m}$ is used in the simulation. Several crack depths $b=50 \mu \mathrm{m}, 100 \mu \mathrm{m}, 150 \mu \mathrm{m}, 200 \mu \mathrm{m}, 250 \mu \mathrm{m}$ are studied. For each crack depth, the crack numbers investigated are 0 and $\mathrm{n}=1,2,3,5,10,15$, i.e., the corresponding crack intervals are infinite and $\mathrm{v}=3 \mathrm{~mm}, 1.5 \mathrm{~mm}, 1 \mathrm{~mm}, 0.6 \mathrm{~mm}, 0.3 \mathrm{~mm}, 0.2 \mathrm{~mm}$.

Fig. 5(a) demonstrates the relationship of crack parameters and the fast heating time $\left(\mathrm{t}_{\mathrm{f}}\right)$ for reaching $450{ }^{\circ} \mathrm{C}$, including the crack number and crack depth. The results indicate that, the $t_{f}$ is shorter and shorter along with the increase of crack depth. What's more, the declined tendency is aggravated for larger numbers. It's obviously that, more areas are involved in the absorption of laser energy, since the surface and in-depth propagation of cracks induced by the repetitive heating. The maximum $t_{f}$ of $60 \mathrm{~s}$ is obtained when there is only one crack of $50 \mu \mathrm{m}$ in the irradiated center, while the minimum $\mathrm{t}_{\mathrm{f}}$ of $0.8 \mathrm{~s}$ is related to the case for 15 cracks of $250 \mu \mathrm{m}$ depth. The value corresponding to the crack number of 0 is also shown for comparison. Once the amount and the depth of cracks increase, the effect of cracks on the thermal process is remarkable.

Fig. 5(b) shows the relationship of crack parameters and the temperature oscillation range $(\Delta T)$, which is induced by the same laser output energy. The $\Delta T$ rises since the cracks depth increases, and this tendency keeps up with the increasing crack numbers, which can be seen from different curves in the picture. Although irradiated by the same laser energy during the same time period, higher temperature range is induced due to higher absorptivity, i.e. more cracked areas are produced owing to surface propagation and in-depth propagation. The maximum $\Delta T$ of $73^{\circ} \mathrm{C}$ is occurred in the case of 15 cracks of $250 \mu \mathrm{m}$ depth, and the minimum $\Delta T$ of $7^{\circ} \mathrm{C}$ is corresponding to the slightest damage situation of 1 crack with $50 \mu \mathrm{m}$ depth, except for the uncracked condition.

The experimental observation of surface cracks after different cycles is presented in Fig. 6. Under the large instantaneous thermal gradient, cracks initiate at the tips of vermicular graphite on the heating surface easily even after the 2rd cycle (shown in Fig. 6(a)). As the test going, the cracks propagate along the graphite phases both on the surface and in the thickness direction. As shown in the Fig. 6(b), after 40 cycles, the change of crack number is not obvious, even though the total crack length increases significantly. Therefore, when simulating the temperature curves, the crack numbers is assumed to be constant, and only the evolution of crack depth is considered. The crack interval can be calculated from the surface distribution, with the minimal value of about $300 \mu \mathrm{m}$, i.e. the crack numbers is 10 .

Based on the above analysis, the temperature histories at different crack conditions is simulated and compared with the measured results. Fig. 7(a) demonstrates the measured temperature response of the surface center during the 2 rd cycle. The fast heating stage changes from $100{ }^{\circ} \mathrm{C}$ to $450^{\circ} \mathrm{C}$ in $19 \mathrm{~s}$, and after that, the laser parameters are transferred to the second setting due to the designed temperature closedloop control mode. Therefore, a temperature oscillation around $450{ }^{\circ} \mathrm{C}$ is inherently superimposed on the existed cycles. From the insert graph in Fig. $7(\mathrm{a})$, the $\Delta T$ is about $35^{\circ} \mathrm{C}$. The calculated temperature-time curve utilizing the proposed model is shown in Fig. 7(b), with the crack characteristics of $\mathrm{b}=50 \mu \mathrm{m}, \mathrm{n}=10$ applied. The simulated result shows almost the same tendency as the measured one, and the simulated $\Delta T$ is also $35^{\circ} \mathrm{C}$. There is good agreement between the experimental and simulated results, and the model validity is verified. As the cracks propagation, the $t_{f}$ diminishes and the $\Delta T$ increases. The measured temperature-time curve of the 20th cycle is presented in Fig. 7(c), showing a smaller $t_{f}$ of $9 \mathrm{~s}$ and larger $\Delta T$ of $53^{\circ} \mathrm{C}$. In order to reflect the temperature changes accurately, the crack parameters applied in the model is modified to $\mathrm{b}=150 \mu \mathrm{m}, \mathrm{n}=10$, and the corresponding result is exhibited in Fig. $7(\mathrm{~d})$. The calculated $\Delta T$ is $55^{\circ} \mathrm{C}$, showing consistency with the actual experiment. The measured temperature-time curve of the 40th cycle is presented in Fig. 7(e), while the value of $\mathrm{t}_{\mathrm{f}}$ and $\Delta T$ is $8 \mathrm{~s}$ and $60^{\circ} \mathrm{C}$ respectively. Accordingly, the crack parameters are changed 

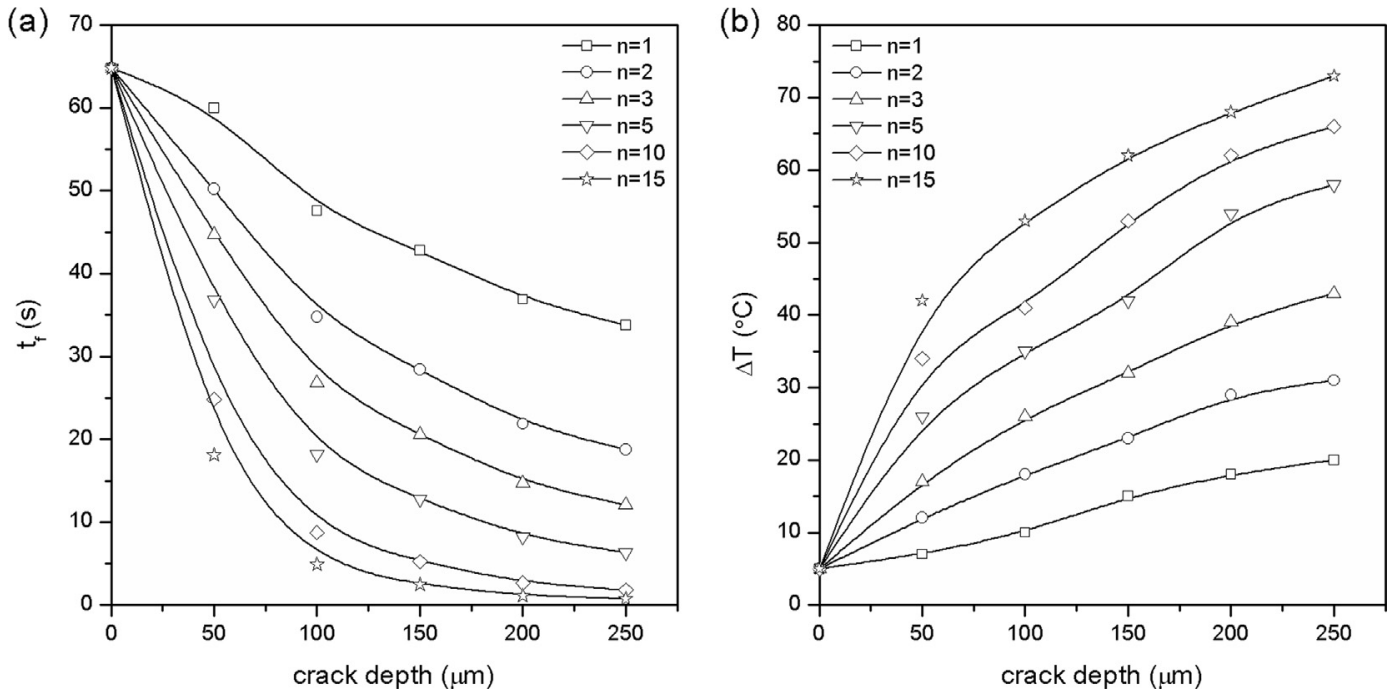

Fig. 5. Relationship of crack depth and the thermal indictors. (a) $t_{f}$; (b) $\Delta T$.

to $\mathrm{b}=200 \mu \mathrm{m}, \mathrm{n}=10$ in the simulation, and the result is shown in Fig. 7(f), and the calculated $\Delta T$ is $60^{\circ} \mathrm{C}$.

During the test, the surface is oxidized severely, causing the decarburization of surface graphite and the formation of oxidation hole. At the bottom of the oxidation holes, which are the stress concentration areas in the microstructure, thermal cracks nucleate and propagate along the depth direction. In order to compare the crack parameters determined by the model with the one obtained by the test, the crack depth after different cycles is observed. Considering the destructiveness of cross-sample preparation, a series of samples are employed for experiments with different cycles, and then the in-depth evolution can be demonstrated in Fig. 8. Although the distribution of graphite in each sample is different, the crack depth after various cycles is still obvious. From Fig. 8(a), a crack with the depth of about $50 \mu \mathrm{m}$ is depicted, which is tested after 2 cycles. The measured result is almost the same with the parameter applied in the simulation of Fig. 7(b). With the testing going, the cracks propagate continually along the thickness direction. The depth is reaching about $150 \mu \mathrm{m}$ after 20 cycles, as revealed in Fig. 8(b). As the oxidation proceeds gradually, the oxygen keeps diffusing inward, causing deeper cracks. After 40 cycles, a crack with nearly $200 \mu \mathrm{m}$ depth is found (in Fig. 8(c)), showing the consistent result with the simulated parameters in Fig. 7.

\section{Discussion}

Despite of the difficulties to detect an actual combustion chamber component, this paper represents a good validation of the proposed simulation model to laboratory experiments, which is tested under the similar thermal working conditions of real components. However, due to the limited heat affect depth of pulsed laser heating, the induced crack depth is strongly dependent on the temperature distribution within the substrate. The simulated temperature distributions of 40th cycle at different times using the parameters of Fig. 7(f) is illustrated in Fig. 9. Fig. 9(a) shows the temperature along the distance from the center on the surface at the time of $7.958 \mathrm{~s}, 8 \mathrm{~s}, 257.76 \mathrm{~s}, 258 \mathrm{~s}$ and $338 \mathrm{~s}$, which is corresponding to the moment of the last pulse of first stage, the end of first stage, the last pulse of second stage, the end of second stage and the end of cooling stage respectively. The temperature within laser irradiated region at $7.958 \mathrm{~s}$ and $257.76 \mathrm{~s}$ is consistent with super-Gaussian distribution of laser energy on the surface. Once the pulse laser is off, i.e. $8 \mathrm{~s}$ and $260 \mathrm{~s}$, the surface temperature redistributes attributed to the heat transfer. Fig. 9(b) shows the temperature along the depth from surface at the center spot. When pulsed laser heating is applied, severe thermal gradient is induced along the thickness direction. The simulated results indicate that the transient fluctuation occurs only within a thin layer below the surface. This layer is defined as the "interaction depth" at which obvious temperature discrepancy occurs [21], which is strongly dependent on the thermal boundary conditions. The temperature gradient caused by the LCF thermal loading is larger than the HCF one, thus producing a larger thermal interaction depth.

The dimensionless temperature profiles and heat flows can be expressed by the dimensionless Fourier number, which compares a characteristic body dimension 1 with an approximate temperature-wave penetration depth for a given time $\tau$.
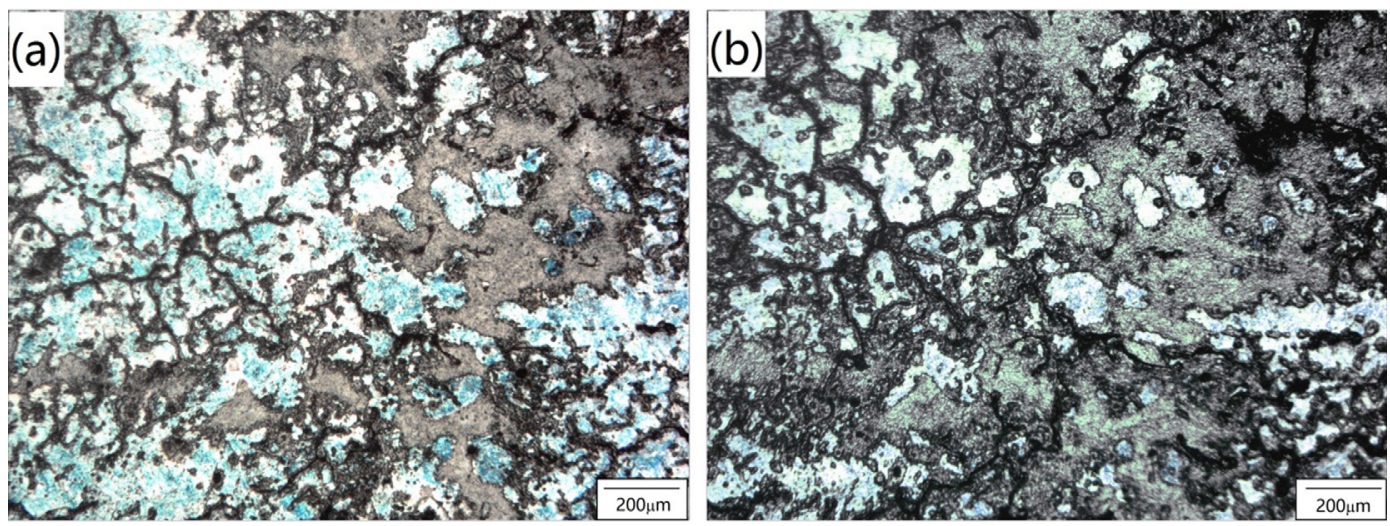

Fig. 6. Surface crack in the irradiated center. (a) after 2 cycles (with $2 \times 10^{3} \mathrm{HCF}$ cycles superimposed); (b) after 40 cycles (with $4 \times 10^{4} \mathrm{HCF}$ cycles superimposed). 

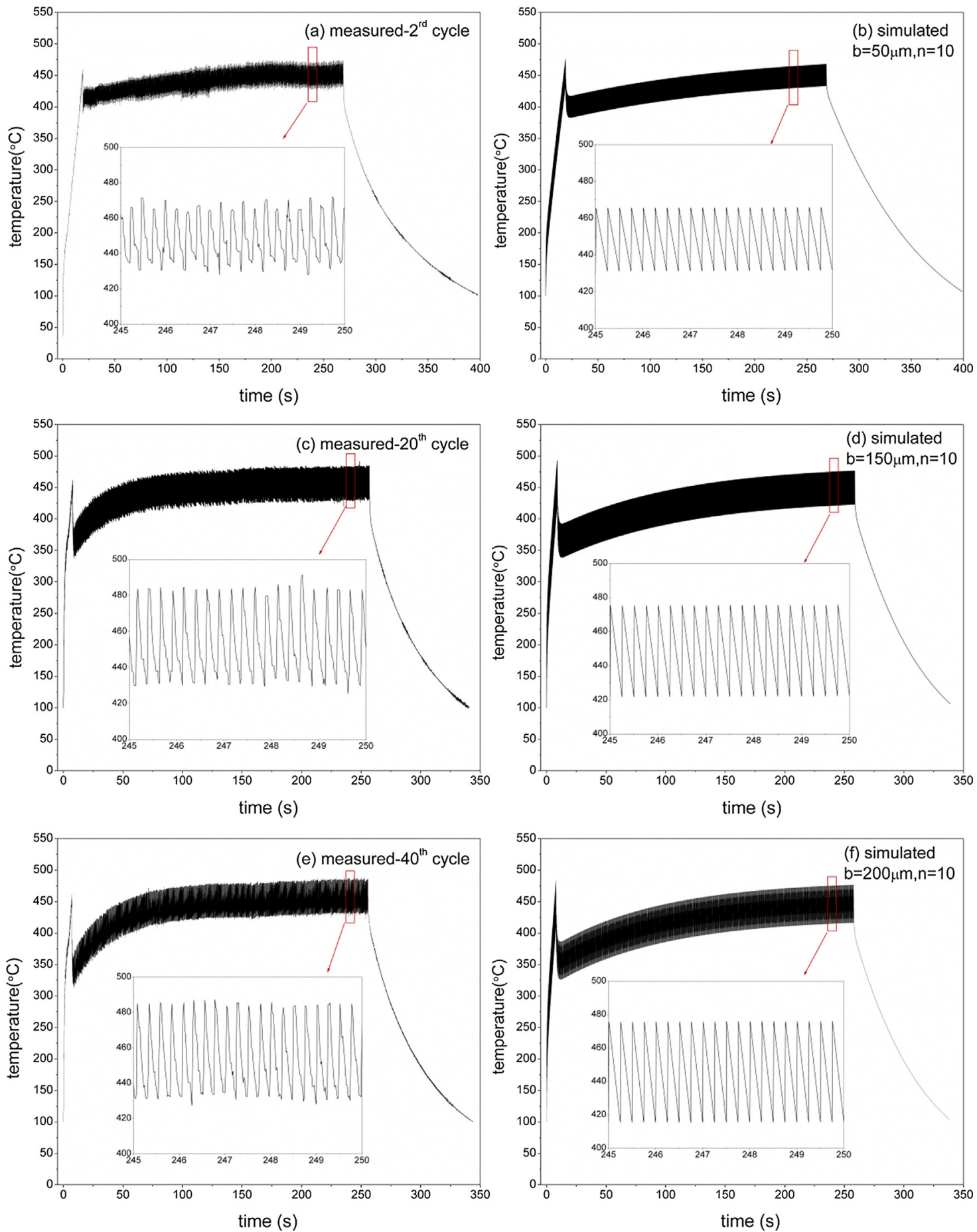

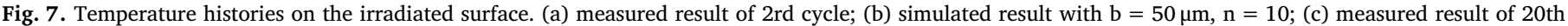
cycle; (d) simulated result with $\mathrm{b}=150 \mu \mathrm{m}, \mathrm{n}=10$; (e) measured result of 40th cycle; (f) simulated result with $\mathrm{b}=200 \mu \mathrm{m}$, $\mathrm{n}=10$.

Fo $=\frac{\tau}{l^{2} / D}$

The model used in this study can be approximately simplified as an infinite plate with a certain thickness, i.e. the 1 value is equal to half of the thickness. The $\tau$ value for the pulsed laser heating is generally defined as the interval of the pulse, for example, $0.25 \mathrm{~s}$ for the pulse of $4 \mathrm{~Hz}$, and $0.05 \mathrm{~s}$ for $20 \mathrm{~Hz}$. The D value means the thermal diffusivity of material, whereas $7.06 \times 10^{-6} \mathrm{~m}^{2} / \mathrm{s}$ for the GJV-450 material. Therefore, the Fo number for the fast heating stage of $20 \mathrm{~Hz}$ and the second stage of $4 \mathrm{~Hz}$ is 0.056 and 0.28 respectively. According to the recent research [22], when the Fo number of infinite plate is larger than
0.24 , the influence of initial condition will disappear, entering into the regular regime of unsteady-state heat transfer. As for the pulsed laser heating, the Fo number is dependent on the pulse interval, once the sample geometry and material is fixed. Considering the analysis above, the $4 \mathrm{~Hz}$ is the critical repetition rate for inducing the stable temperature oscillation, because once the pulse of $4 \mathrm{~Hz}$ is removed, the overall temperature difference along the thickness direction will decrease rapidly and reach quasi-uniform state (see in Fig. 9(b)). If the pulse repetition rate is increased to $20 \mathrm{~Hz}$, i.e. the Fo number is reduced to 0.056 , it will be a large temperature gradient along the thickness direction after the pulse removal (see in Fig. 9(b)). Once the next pulse 


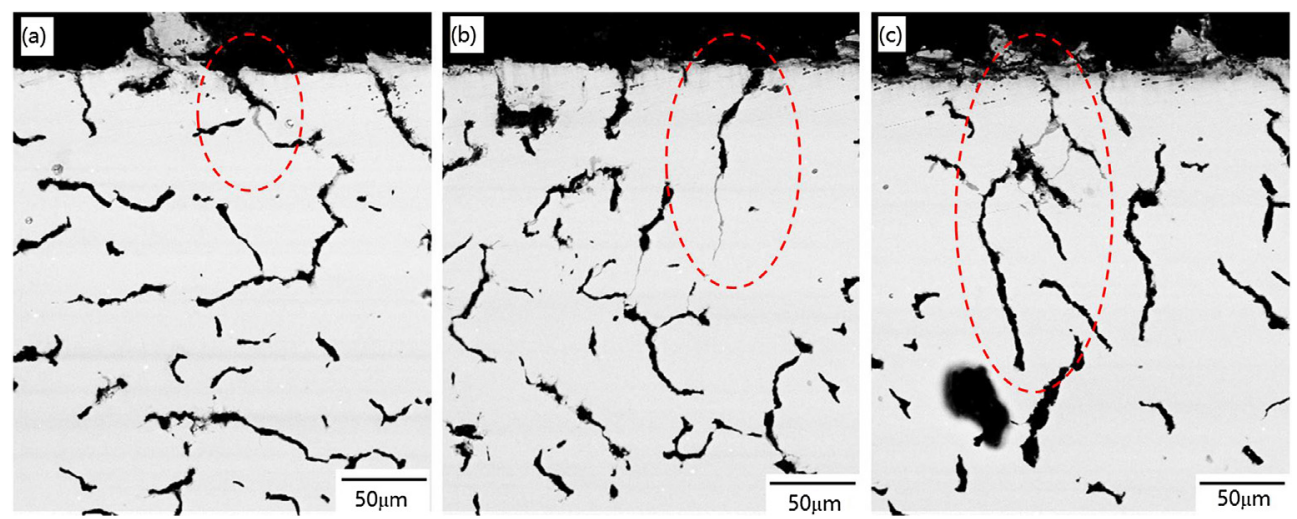

Fig. 8. In-depth crack (SEM) in the irradiated center. (a) after 2 cycles; (b) after 20 cycles; (c) after 40 cycles.
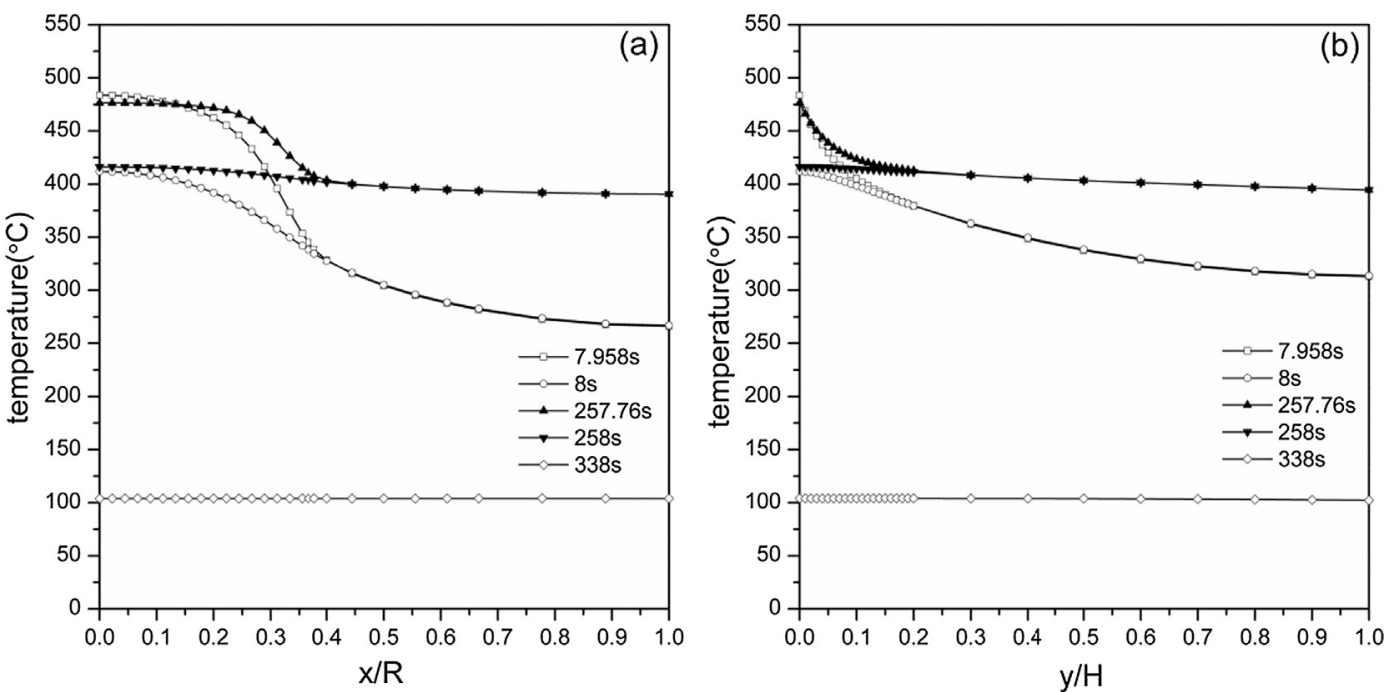

Fig. 9. Simulated results of temperature distributions. (a) along the distance from center on the surface; (b) along the depth from surface at the center spot.
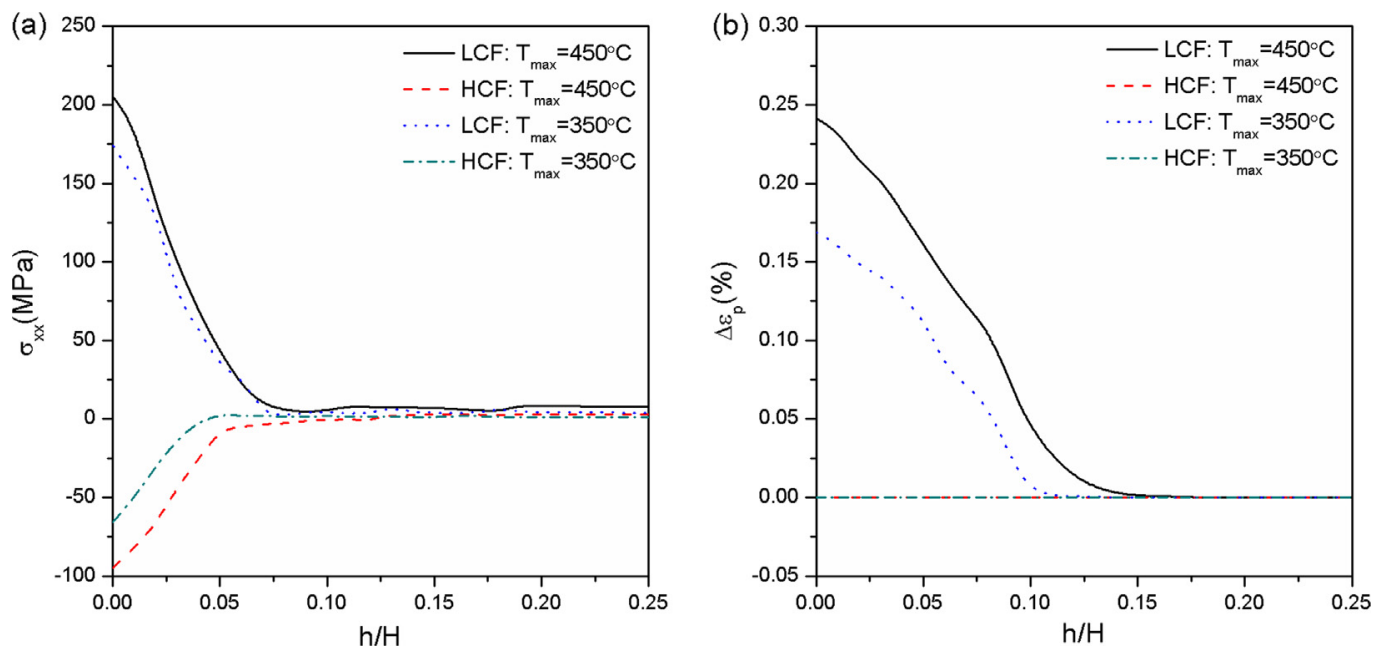

Fig. 10. Simulated results along the depth from surface for 40th cycle. (a) stress; (b) strain.

acts again, the temperature difference keeps increasing, resulting in significant thermal accumulation effect, and showing the similar heating behavior like the quasi-continuous laser.

From the Fig. 9, the frequent temperature fluctuation almost occurs within the surface shallow region, and there is a strong thermal loading gradient in the depth, leading to the crack propagation within a finite thin layer. As demonstrated in Fig. 10, the thermal stress and thermal strain during the CCF test is calculated using finite element method. In comparison, the simulated results for maximum temperature of $350{ }^{\circ} \mathrm{C}$ is also shown in the figure. Fig. 10(a) presents the distribution of the first principle stress along the depth direction. The LCF value shows the maximum on the surface, and decrease to zero at certain depth, while the HCF value obtains the maximal compressive stress on the surface and increases to zero below specific depth. However, the LCF 

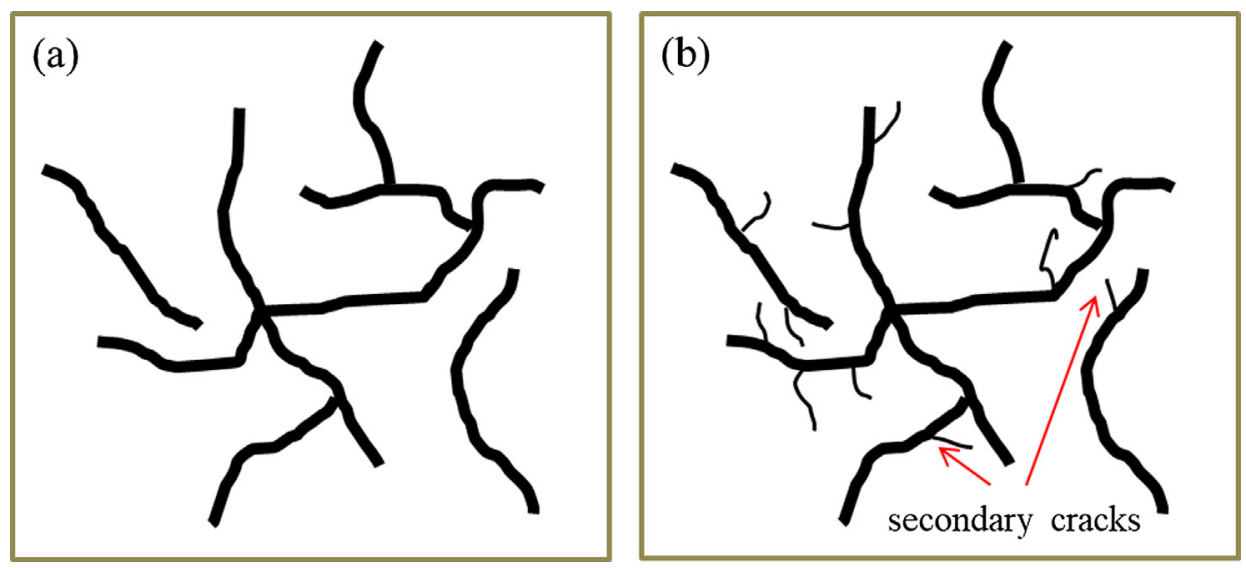

Fig. 11. Schematic diagrams of crack patterns. (a) LCF test; (b) CCF test.

interaction depth of is slightly deeper than the HCF one, due to the larger temperature range and longer interaction time. From Fig. 10(b), considerable plastic stain is accumulated within a finite small thickness during the LCF stage, while almost no plastic strain is occurred in the HCF stage.

As mentioned above, different loadings cause different patterns of crack damage, as demonstrated in Fig. 11 schematically. Compared to the pure LCF tests (see in Fig. 11(a)), the CCF tests (see in Fig. 11(b)) induce more secondary cracks besides several major cracks, forming denser crack networks. It suggests that transient larger temperature gradient is superimposed on the surfaces by the addition of the pulsed HCF oscillation. The mechanisms of thermal cracks initiation under variable amplitude thermal cycles, can be tensile-stress induced cracking in the cooling stage, and peak compressive-stress induced cracking during the HCF heating stage [2]. Since the pulsed laser HCF component would promote the surface compressive cracking, the accelerated crack propagation and higher crack density can be expected. Due to the crack closure effect and the interaction of variable amplitude thermal loadings, the effect of superimposed HCF loadings on the fatigue crack growth is complicated.

The simulated results show that, the large LCF temperature variations are high enough to produce plastic deformation which is necessary for the crack initiation. Once the thermal cracks grow into the specimen, the deformation becomes elastic gradually. Finally, a fully elastic zone without tensile stress is formed, and no further propagation occurs. It is consistent with the fact that the thermal cracks only occur within a thin layer near the specimen surface, and the crack growth along the depth direction is inhibited. Furthermore, the superimposed temperature fluctuation of high frequency on the surface produces cyclic elastic stresses under the yield limit, which is dynamic in nature with a shorter interaction time and smaller interaction depth [2], when compared to the LCF process. The HCF thermal loadings act on the initiated LCF surface crack in a wedging process, and increase the crack tip opening displacement by high frequency cyclic stresses, offering additional driving force of crack propagation. Therefore, the crack length after specific LCF cycle can be expressed as

$\mathrm{a}=\int\left[\mathrm{C}\left(\Delta K_{L C F}\right)^{m}+\sum_{i=0}^{N_{H C F}} \mathrm{C}\left(\Delta K_{H C F}\right)^{m}\right] d N$

where $\mathrm{m}$ and $\mathrm{C}$ are constants, $\mathrm{N}_{\mathrm{HCF}}$ is the number of HCF cycles per LCF cycle, $\Delta \mathrm{K}_{\mathrm{LCF}}$ and $\Delta \mathrm{K}_{\mathrm{HCF}}$ are the stress intensity factor amplitudes under LCF and HCF thermal loadings respectively. The crack growth rate strongly depends on the $\mathrm{N}_{\mathrm{HCF}}$. For $1000 \mathrm{HCF}$ cycles or more, significantly higher crack growth rates could be observed [23-25]. As in this paper, the $\mathrm{N}_{\mathrm{HCF}}$ is selected to be 1000 by setting the heating time of HCF stage to $250 \mathrm{~s}$, and the effect of different HCF cycles on the crack propagation rate will be studied in the future.

\section{Conclusion}

At the present work, the thermal cracks under variable amplitude thermal fatigue is investigated, which are induced by the millisecond pulsed laser innovatively. Furthermore, the temperature field, thermal stress and thermal strain are numerically calculated. The main results are summarized as follows:

(1) The LCF fast heating time and the HCF temperature oscillation range are considered as two typical indictors, and the changes in the thermal indictors are observed as functions of the crack parameters, showing the coupled effect of surface damage state and the subsequent thermal response. The numerical prediction of diverse cracks parameters, are in accordance with the experimental results at different cycles, verifying the accuracy of the proposed model.

(2) The observed limited cracks in-depth can be explained by the obvious discrepancy of temperature and stress within a limited thin layer below the surface. The driving forces of crack propagation under thermal loading of variable amplitude, include the tensilestress in the cooling stage and peak compressive-stress during the HCF heating stage. The addition of pulsed HCF oscillation induces more secondary cracks and forms denser crack networks. The LCF interaction depth of is larger than the HCF one, causing more profound influence under the surface. The LCF loading mainly affects crack initiation, while the effect of superimposed HCF loading is to accelerate crack propagation by a surface wedging mechanism.

(3) The crack damage process of cast iron under variable amplitude thermal loading is discussed experimentally and numerically, which is also applicable in other material working under similar combined thermal conditions, helping for failure analysis and design optimization.

\section{Acknowledgments}

This work was supported by the National Natural Science Foundation of China under grant Nos. 11272316, 11502269, 11672304 and 11272317. In addition, special thanks for the instrument developing project of the Chinese Academy of Sciences (No. yz201636).

\section{References}

[1] Trampert S, Gocmez T, Pischinger S. Thermomechanical fatigue life prediction of cylinder heads in combustion engines. J Eng Gas Turbines Power 2008;130:012806.

[2] Dongming Zhu RAM. Investigation of thermal fatigue behavior of thermal barrier coating systems, Surf Coat Technol. 1997; 94-95: 94-101.

[3] Kutsuna M, Fujita S, Sugita Y, Yamada K. Thermal fatigue test for turbine housing by a pulse YAG laser. High-power lasers in manufacturing. 2000; 3888: 438-45.

[4] Cerdoun M, Carcasci C, Ghenaiet A. An approach for the thermal analysis of internal combustion engines' exhaust valves. Appl Therm Eng 2016;102:1095-108. 
[5] Lu X, Li Q, Zhang W, Guo Y, He T, Zou D. Thermal analysis on piston of marine diesel engine. Appl Therm Eng 2013;50:168-76.

[6] Zhang Q, Zuo Z, Liu J. Failure analysis of a diesel engine cylinder head based on finite element method. Eng Fail Anal 2013;34:51-8.

[7] Mavropoulos GC. Experimental study of the interactions between long and shortterm unsteady heat transfer responses on the in-cylinder and exhaust manifold diesel engine surfaces. Appl Energy 2011;88:867-81.

[8] Wang X-S, Zhang W-Z. Oxidation and thermal cracking behavior of compacted graphite iron under high temperature and thermal shock. Oxid Met 2016;87:179-88.

[9] Wang X, Zhang W, Guo B, Zhao W. The characteristics of microcrack initiation process in cast iron materials under thermal shock test. Mater Sci Eng, A 2014;609:310-7.

[10] Tsuyoshi T, Sasaki K. Low cycle thermal fatigue of aluminum alloy cylinder head in consideration of changing metrology microstructure. Procedia Eng 2010;2:767-76.

[11] Gan Z, Yu G, Li S, He X, Chen R, Zheng C, et al. A novel intelligent adaptive control of laser-based ground thermal test. Chin J Aeronaut 2016;29:1018-26.

[12] Yu G, He X.L., Li S.X. Laser manufacturing and its application, National Defense Industry Press, Beijing; 2016.

[13] Schaus PM. M, Nd-YAG-Laser simulated thermal shock and thermal fatigue behaviour of railroad steel. Metall 1998;52:464-70.

[14] Wang M, Li Y, Wang Z, Bao E. Effect of rare earth elements on the thermal cracking resistance of high speed steel rolls. J Rare Earths 2011;29:489-93.

[15] Long SG, Zhou YC. Thermal fatigue of particle reinforced metal-matrix composite induced by laser heating and mechanical load. Compos Sci Technol 2005;65:1391-400.
[16] Vincent L, Poncelet M, Roux S, Hild F, Farcage D. Experimental facility for high cycle thermal fatigue tests using laser shocks. Procedia Eng 2013;66:669-75.

[17] Wang Y, Shen N, Befekadu GK, Pasiliao CL. Modeling pulsed laser ablation of aluminum with finite element analysis considering material moving front. Int $\mathrm{J}$ Heat Mass Transfer 2017;113:1246-53.

[18] Pan S, Yu G, Li S, He X, Xia C, Ning W, et al. Application of millisecond pulsed laser for thermal fatigue property evaluation. Opt Laser Technol 2018;99:382-91.

[19] Sihn S, Childers LB, Walters CT, Forte MS, Roy AK, Vernon JP. Computational and experimental study on laser heating of a Ni-based metal alloy. Int J Heat Mass Transfer 2016;102:1034-43.

[20] Paffumi E, Nilsson K-F, Szaraz Z. Experimental and numerical assessment of thermal fatigue in 316 austenitic steel pipes. Eng Fail Anal 2015;47:312-27.

[21] Dongming Zhu RAM. Investigation of thermal high cycle and low cycle fatigue mechanisms of thick thermal barrier coatings, Mater Sci Eng A. 1998; 245: 212-223.

[22] S.H. Grigull U. Heat conduction, Hemisphere Publishing Corporation, Washington; 1984.

[23] Schweizer C, Seifert T, Nieweg B, von Hartrott P, Riedel H. Mechanisms and modelling of fatigue crack growth under combined low and high cycle fatigue loading. Int J Fatigue 2011;33:194-202.

[24] Hosseini E, Holdsworth SR. Cracking due to combined TMF and HCF loading in cast iron. Int J Fatigue 2017;99:279-85.

[25] Norman V, Skoglund P, Leidermark D, Moverare J. Damage mechanisms in siliconmolybdenum cast irons subjected to thermo-mechanical fatigue. Int J Fatigue 2017;99:258-65. 